\title{
VAC.II - Developing an intranasal vaccine against canine visceral leishmaniasis: a study of efficacy in mice
}

\author{
Izabella Pereira da Silva Bezerra ${ }^{1 *}$; Bartira Rossi-Bergmann ${ }^{1}$. \\ 1UFRJ/Instituto de Biofísica Carlos Chagas Filho.
}

Introduction: Visceral leishmaniasis (VL) is caused in America and in Europe by L. infantum and is lethal if not treated. Although dogs are the main domestic reservoirs, available canine vaccines are administered only after 4 months of age, leaving younger puppies unprotected. Our group has developed a tolerogenic strategy based on mucosa vaccination to prevent the early Th2 counter-protective response elicited by the infecting parasite. Indeed, intranasal (i.n.) vaccination with whole Leishmania amazonensis antigens ( $\mathrm{LaAg}$ ) has proven to protect mice and hamsters against both cutaneous and VL, showing a broad spectrum of action. Moreover, retinoic acid (a vitamin A metabolite) encapsulated in nanoparticles (RA-NP) acts as an adjuvant for i.n. LaAg, increasing protection in BALB/c mice against L. amazonensis and in hamsters against L. braziliensis infection by enhancing $\mathrm{CD} 4^{+} \mathrm{Foxp}^{+} \mathrm{T}^{\mathrm{reg}}$ population in nasal mucosa draining lymph nodes. Besides being needle-free, an i.n. vaccine could be administered in dogs from 3 weeks of age, conferring earlier protection.

Objective: Based on these findings, we proposed to evaluate the LaAg/RA-NP i.n. vaccine efficacy against $L$. infantum infection in mice, aiming at the development of an innovative i.n. vaccine against canine VL.

Methodology: For comparative evaluation, BALB/c young ( 8 weeks-old) or newborn (10 daysold) mice were immunized with 2 i.n. doses of $\mathrm{LaAg} / \mathrm{RA}-\mathrm{NP}$ with a 7 day-interval between them. Controls were not vaccinated or received 3 s.c. doses of the marketed Leish-Tec ${ }^{\circledast}(10$ $\mu \mathrm{g}$ protein $+50 \mu \mathrm{g}$ saponin/dose) with a 7-day-interval. Seven days after immunization, Leishmania-specific antibodies were quantified in serum and animals were intravenously challenged with $L$. infantum promastigotes $\left(2 \times 10^{7}\right)$. Seven days after infection, transcription factor and cytokine expression in spleen were evaluated by quantitative real-time PCR and 35 days after infection parasite loads were evaluated in liver and spleen by limiting dilution assay.

Results: As Leish-Tec ${ }^{\circledast}$, our vaccine does not induce antibodies that interfere with the detection of active infection, using serum from infected animals as positive control. During early infection, LaAg/RA-NP modulates expression of transcription factors and cytokines in spleen, increasing, mainly, IL-10 expression, suggesting a peripheral suppressive response. We found that LaAg/RA-NP is more effective than Leish-Tec ${ }^{\circledast}$, reducing $94 \%$ of the parasite load in the spleen and $91 \%$ in the liver compared to the non-immunized group against $54 \%$ and $82 \%$ reduction promoted by the latter. In addition, LaAg/RA-NP proved to be effective in newborn mice, reducing the parasite load in the spleen $(75 \%)$ and liver (81\%) compared to non-immunized animals.

Conclusion: Therefore, our results demonstrate that $\mathrm{LaAg} / \mathrm{RA}-\mathrm{NP}$ is a promising vaccine to be tested against canine VL particularly aimed at newborn animals.

Keywords: Canine visceral leishmaniasis; intranasal vaccine; mucosa 